\title{
Psychosocial rehabilitation in a chronic care hospital in South Africa: views of clinical staff
}

\author{
AL Pillay, AL Kramers-Olen \\ Department of Behavioural Medicine, Nelson R Mandela School of Medicine, University of KwaZulu-Natal, Durban, KwaZulu-Natal, \\ South Africa
}

\begin{abstract}
Objective: The study was based at a South African hospital providing inpatient care for people with chronic mental disorders, and aimed at investigating the multidisciplinary team (MDT) members' views, understanding and attitudes towards psychosocial rehabilitation (PSR). Method: A survey method was used, with the MDT members completing a questionnaire that tapped, inter alia, their understanding of PSR, perceived aims and goals of PSR, adequacy of their professional training to implement PSR, availability of resources to implement PSR, and their views of chronic mentally ill patients, including their functional skills, needs and prognosis. Results: Of 114 respondents, 19.3\% felt they had sufficient knowledge of PSR practice, 53.5\% reported that their professional training included teaching on PSR, 90.4\% wanted to undertake further training in PSR, 64.9\% believed that the hospital environment lends itself to PSR, and 23.7\% felt the hospital had sufficient resources to perform PSR. Most (93\%) believed that patients with chronic mental disorders can improve their functioning, and 65.8\% thought that such patients could make decisions for themselves. Conclusion: It is not surprising that most of the clinical staff in this investigation do not feel sufficiently equipped to perform PSR interventions, considering that past mental health policies and training failed to emphasize this component of mental health care. The need for training programmes to address this deficit is evident.
\end{abstract}

Keywords: Psychosocial rehabilitation; Mental disorder; Mental illness; Ppsychosis; Mental health

Received: 05-09-2012

Accepted: $28-09-2012$

doi: http://dx.doi.org/10.4314/ajpsy.v16i4.37

\section{Introduction}

The past three decades has seen the development of a substantial body of research evidence demonstrating the efficacy of psychosocial interventions for persons disabled by mental health problems. The benefits of psychosocial interventions in combination with optimal pharmacological treatments are widely accepted, along with treatment and rehabilitation approaches that are cognisant of the phase of the mental disorder. ${ }^{1}$ Furthermore, there is evidence to suggest that persons with psychotic disorders like schizophrenia who are involved in psychosocial rehabilitation programmes may need lower doses of medication. ${ }^{2}$

The ability of people with severe mental illnesses to perform the basic tasks and roles of daily living is often compromised. ${ }^{3}$ This problem, together with the long term disability experienced by persons with severe and persistent mental disorders, has

\section{Correspondence}

Dr AL Pillay

Fort Napier Hospital, PO Box 370, Pietermaritzburg, 3200, South Africa

email: anthony.pillay@kznhealth.gov.za informed the need for psychosocial rehabilitation programmes that have a greater focus on long-term and inclusive rehabilitation interventions. ${ }^{4}$ Moreover, there is an urgent need for poorer countries to take seriously the concept of rehabilitation of the severely mentally ill and implement such programmes in their mental health services.

While psychosocial rehabilitation (PSR) has previously been inconsistently defined in the literature, it is accepted to refer to a combination of occupational, social, educational, behavioural and cognitive interventions aimed at increasing the quality of life of those affected with serious and persistent mental illness. ${ }^{5}$ Psychosocial rehabilitation interventions have also received increasing attention in line with policies of de-institutionalization and community based care models, with a greater focus on selfsufficiency and long term recovery. Wallace, Liberman, Kopelowicz and Yaeger ${ }^{6}$ lend support to previous suggestions that standards of care for persons with severe and persistent mental illness should include interventions in clinical areas (symptom reduction); rehabilitative interventions (improving vocational and social functioning); humanitarian interventions as well as 
interventions in the area of public welfare. The World Health Organization's mhGAP Intervention Guide ${ }^{7}$ also advocates that persons with psychotic disorders must be encouraged to resume their social, occupational and educational activities as a way of facilitating their inclusion in the social, cultural and economic contexts. Similarly, Kopelowicz, et $\mathrm{al}^{8}$ posit that a holistic approach to PSR must attempt to realistically restore the level of functioning of a person disabled by mental illness in the areas of social, vocational, work, family, leisure, friendship and spiritual domains. The ideal scenario includes the active participation of the patient in the process of goal setting and treatment planning, with the least amount of professional intervention. In this context of active involvement, the PSR treatment mall concept has been documented as a practical and efficient way of rehabilitating where patients (and staff) move out of their units to a central area where they participate in the rehabilitation programme. ${ }^{9}$ In a similar philosophy, the recovery model also emphasizes the role of personal responsibility, and recognises that patients must be actively involved in their recovery process..$^{10}$ Mellsop and Diesfeld have added that the rights and autonomy of patients must be maximized, while acknowledging the need for degrees of autonomy rather than total autonomy. ${ }^{10}$

Almost two decades ago, Liberman and Corrigan ${ }^{11}$, in their description of barriers to the implementation of inpatient behavioural interventions, cited the deleterious effects of insufficient human resources, inadequately trained staff, philosophical opposition to programme implementation, and administrative red tape and bureaucracy. Unfortunately, this is still applicable, with most of these factors continuing to hinder the provision of appropriate rehabilitation services to those with severe and chronic mental disorders. In poorer countries limited mental health funding inevitably means prioritising acute mental health problems. As a result, chronic conditions such as schizophrenia and other debilitating conditions receive inadequate funding. This is particularly worrisome considering $80 \%$ of those afflicted with schizophrenia live in middle- and lowincome countries. ${ }^{12}$ Furthermore, such individuals in chronic stages of the illness are often institutionalised, and unless they are effectively rehabilitated they cannot realistically be discharged to their communities. Not only does this increase national costs and disease burden it is also contrary to the goals of the community mental health agenda. The deinstitutionalization argument has been further supported by the assertion that, compared to institution based care, community mental health services can be more effective in caring for the needs of those with severe mental disabilities, and possibly pose less risk for neglect and human rights violations. ${ }^{13}$

A further problem relating to the development and implementation of PSR programmes in institutions is the extent to which mental health professionals are trained in this type of intervention. A recent report from the United States (US) cited staff training as a significant barrier to the implementation and transformation of a recovery-oriented system ${ }^{14}$ for people with severe mental disorders. A national European study noted that training in rehabilitation is limited with less than half of their nationally surveyed psychiatrists reporting the provision of such training. ${ }^{15}$ Yet a recent clinical trial in China found that a PSR programme implemented by staff who had recently received specific training resulted in significant reductions in psychopathology symptoms and an improvement in patients' attitude towards medication. ${ }^{2}$ In addition to clinical benefits, a recent investigation has shown that appropriate training to equip staff for the demands of a psychiatric rehabilitation setting can enhance cohesiveness in the workforce. ${ }^{16}$ Research has also demonstrated the significance to developing competence in the context of the various socio-cultural issues that need to be embedded into PSR programmes in order to ensure their effectiveness. ${ }^{17}$ This is especially relevant in multicultural South Africa.

The need for a multidisciplinary approach to PSR cannot be sufficiently emphasized considering the various types of input required to optimise overall functioning within the individual and his/her relationships with others. A recent investigation in Belgium noted that their rehabilitation programmes were generally provided by multidisciplinary teams (MDT's), although they noted that the size of the teams varied according to the type of facility, with psychiatric hospitals having larger teams. ${ }^{15}$ This is not surprising considering that the need to develop conjoint interventions amongst the multi-disciplinary team members is central to the effective implementation of psychosocial rehabilitation. ${ }^{18}$ The World Health Organization ${ }^{19}$ has also argued the value of MDT's in view of the complex needs of patients and their families. Unfortunately, a common scenario in mental health facilities is that of professional disciplines operating in isolation. ${ }^{20}$

\section{Context}

The current research was conducted at a major inpatient mental health facility in South Africa, designated for long-term patients with chronic mental health problems, as well as State Patients. The latter are individuals with mental health problems, under courtordered detention in a mental health facility following their involvement or alleged involvement in criminal activities. The facility currently cares for approximately 250 inpatients.

Historically the facility under study was designated a psychiatric hospital for acute mental disorders, specifically for Black patients. As a function of the prevailing apartheid policies and structures, the facility was in a rather dilapidated state with extremely old, poorly maintained buildings that were remnants of the British colonial military era in South Africa. The American Psychiatric Association's ${ }^{21}$ report on the mental health facilities for Black patients detailed the state of some of these structures. Since the inception of democracy the hospital has been re-designated as a long-term mental health facility, and serves people of all race groups. With the political change some improvement and refurbishment of the facility has taken place, but there is still much to be desired in terms of physical facilities, especially for the care of persons with mental health problems.

With its renewed mental health purpose the institution has been on a quest to develop an appropriate, structured programme that could best serve its rehabilitative function. To this end the MDT has embarked on a mission of establishing a PSR programme that could serve the dual purposes of (i) rehabilitating patients to the point of improving their adaptive functioning and achieving optimal adjustment, and (ii) preparing patients for discharge from the hospital and re-integration into the community. In this context the authors became aware that there was not a common understanding of the idea of rehabilitation and the term psychosocial rehabilitation among the MDT members. Recent South African research has also noted that PSR services have not been well developed in the country, with policy development issues hampering the process. ${ }^{22}$

The need to survey the ideas and views of the mental health 
professionals on this issue was evident, because attempts to introduce a PSR programme into the facility would be futile if there was no uniform understanding of the concept, its aims, and practices..$^{23}$ In addition, it was considered valuable to have a baseline assessment of staff knowledge and awareness of PSR before training on the programme could begin.

\section{Method}

\section{Participants}

The population for this study comprised all of the clinical staff (i.e. psychiatrists, medical doctors, nurses, clinical psychologists, social workers, occupational therapists and pharmacists) at the hospital. All of the staff were approached for participation and those that agreed constituted the sample.

\section{Instrument}

In view of the unique issues surrounding the specific hospital context, the authors considered it useful to construct a questionnaire that would tap the areas they were interested in investigating. In addition to basic demographic and occupational details the following questions were asked:

(1) Is language a challenge in working with your patients?

(2) Should one discipline be mainly responsible for PSR?

(3) Do you feel you have sufficient knowledge in PSR practice?

(4) Did your professional training include teaching on PSR?

(5) Do you receive any in-service training on PSR in your current work context?

(6) Do you believe there should be further formal training on PSR?

(7) Would you want to undertake further formal training specifically on PSR?

(8) Do you think students in your field should be taught PSR in their basic professional training?

(9) Does the hospital environment lend itself to PSR?

(10) Does the hospital have sufficient resources to do PSR?

(11) Are there sufficient step-down / half-way houses in the community for when your patients are discharged

(12) Do you think chronic mentally ill patients can improve in their functioning?

(13) Do you think chronic mentally ill patients can make decisions about their life?

(14) Do you think the patient's family has a role in PSR?

(15) Do your patients have sufficient contact with their families?

(16) Do you think patients with Mental Retardation can benefit from PSR?

(17) Do you think patients who have had Schizophrenia for many years can benefit from PSR?

Questions were generally structured in a quantitative format, with most requiring a 'yes' or 'no' response.

\section{Procedure}

Prior to beginning the field work the research idea was discussed with the hospital management who gave the investigation their support. To this end the institutional clinical staff were informed about the investigation and that they would be approached for participation. Participants were recruited either (i) just prior to a staff meeting when they were in a large group, or (ii) in their respective wards and units of work. In both settings staff were informed about the research and its purpose, and given copies of the questionnaire, information sheet and consent form, for completion should they choose to participate. The staff were requested not to write their names on the questionnaires, but to ensure anonymity a second level strategy was employed. The potential participants were requested to fold the completed and uncompleted questionnaires and deposit them into a container that was provided.

\section{Data analysis}

The data were tabulated and analysed using the Statistical Package for the Social Sciences (SPSS 19).

\section{Ethics}

Ethical approval for the investigation was obtained from the authors' institutions i.e. University of Kwa Zulu Natal and Fort Napier Hospital.

\section{Results}

A total of 114 completed questionnaires were received, which constituted the sample for this investigation. A response rate of 40.7\% was achieved, considering the total clinical staff in the institution is 280 , although a small number may have been absent at the time of the investigation.

Of the participants 69 (60.5\%) were women and 42 (36.8\%) were men, with 3 (2.6\%) missing data. The gender distribution was considered reasonably representative. The mean age was 37.2 years (s.d. = 9.36) with a range between 21 and 58 years, although there were 8 missing data. The professional distribution of the participants was as follows: 95 (83.3\%) nursing, 9 (7.9\%) clinical psychology, 4 (3.5\%) occupational therapy, 1 medical/psychiatry and 1 social work. There was a slight overrepresentation of clinical psychologists and occupational therapists, with a slight under-representation of medical/psychiatry and social work staff. Considering the small numbers of these staff in the institution, this result was not considered a serious problem. For the 83 (72.8\%) participants who indicated the number of years working with chronic mentally ill patients, the range was 1 to 40 years with a mean of 9.4 years (s.d. = 9.38).

Table I presents the results to questions requiring a 'yes' or 'no' response. Regarding the proportion of time that should be spent on PSR in basic training, 64 (56.2\%) participants believed it should be over $20 \%$. While only half (57) the sample responded to the question asking what would contribute most to improving their patients' functioning, 42 (73.7\%) cited skills training. Gender had no significant effect on most variables, except that significantly more of the respondents who believed their patients had sufficient contact with their families were men $\left(\chi^{2}=6.93\right.$, d.f. $\left.=1, p=0.008\right)$. In addition, among those participants who felt the hospital did not have sufficient resources to perform PSR significantly more were women $\left(\chi^{2}=4.47\right.$, d.f. $\left.=1, p=0.035\right)$.

Among those whose professional training included teaching in PSR, significantly more reported getting satisfaction from working with chronic mentally ill patients $\left(\chi^{2}=4.76\right.$, d.f. $\left.=1, p=0.029\right)$. Approaching significance were the findings that among those wanting training in PSR more were satisfied with their work with the chronic mentally ill ( $\chi^{2}=3.49$, d.f. $\left.=1, p=0.062\right)$, and among those who reported receiving in-service training in PSR more were satisfied with their work with the chronic mentally ill $\left(\chi^{2}=\right.$ 2.85, d.f. $=1, \mathrm{p}=0.092$ ).

Of those reporting receiving no in-service training in PSR significantly more reported not having language challenges in 
Table I: Selected results

\begin{tabular}{|c|c|c|c|}
\hline & n & $\%$ & Missing data ( $n$ ) \\
\hline Satisfied working with chronic patients & 90 & 78.9 & 5 \\
\hline Language is a challenge & 37 & 32.5 & 6 \\
\hline One discipline should be responsible for PSR & 27 & 23.7 & 3 \\
\hline Have sufficient knowledge in PSR practice & 22 & 19.3 & 5 \\
\hline Professional training included teaching on PSR & 61 & 53.5 & 2 \\
\hline Receive in-service training on PSR in current work context & 29 & 25.4 & 1 \\
\hline There should be further formal training in PSR & 107 & 93.9 & 1 \\
\hline Want to undertake further formal training specifically on PSR & 103 & 90.4 & 4 \\
\hline Students in your field should be taught PSR in their basic professional training? & 109 & 95.6 & 1 \\
\hline The hospital environment lends itself to PSR & 74 & 64.9 & 4 \\
\hline The hospital has sufficient resources to implement PSR & 27 & 23.7 & 6 \\
\hline There are sufficient step-down facilities in the community for when patients are discharged & 16 & 14.0 & 7 \\
\hline Chronic mentally ill patients can improve in their functioning & 106 & 93.0 & 2 \\
\hline Chronic mentally ill patients can make decisions about their lives & 75 & 65.8 & 6 \\
\hline The patient's family has a role in PSR & 102 & 89.5 & 2 \\
\hline Patients have contact with their families & 34 & 29.8 & 7 \\
\hline Patients with Mental Retardation can benefit from PSR & 94 & 82.5 & 7 \\
\hline Patients who have had Schizophrenia for many years can benefit from PSR & 106 & 93.0 & 3 \\
\hline
\end{tabular}

communicating with patients $\left(\chi^{2}=4.12\right.$, d.f. $\left.=1, p=0.043\right)$ and not having sufficient knowledge in PSR practice $\left(\chi^{2}=8.34\right.$, d.f. $=1, p=$ 0.004). Among those who felt they had sufficient knowledge in PSR practice more reported that their professional training included PSR teaching ( $\chi^{2}=3.65$, d.f. $\left.=1, \mathrm{p}=0.056\right)$.

Of those participants who believed their patients have insufficient contact with their families, significantly more felt the hospital had insufficient resources to do PSR $\left(\chi^{2}=16.42\right.$, d.f. $=1, \mathrm{p}$ $=0.0005$ ). Significantly more of those participants who reported insufficient PSR knowledge believed their patients had insufficient contact with their families $\left(\chi^{2}=4.39\right.$, d.f. $\left.=1, p=0.036\right)$.

\section{Discussion}

The predominance of women in the sample is understandable, given that the population from which the sample was drawn comprised mainly nurses, among whom women feature prominently in all parts of the world. ${ }^{24}$

The finding that almost $80 \%$ of the respondents were satisfied with their work with chronic psychiatric patients is encouraging for mental health services, considering more satisfied staff results in less staff turnover. ${ }^{25}$ This is important in creating a stable therapeutic milieu if the PSR programme is to be successful. In addition, the finding affirms of the institution's employment selection processes, especially since the care of the chronic mentally ill is not a career preference for many mental health professionals. Research among clinical psychologists, for example, revealed a preference for private practice, providing acute care to the more affluent. ${ }^{26}$

Language was noted as a challenge in almost one-third of the respondents. This relates to the multicultural nature of South African society where there are 11 official languages. While it is important that skills in the major languages within geographical work areas are acquired, it will be a while before the ideal is reached. The challenges of language in mental health care is prevalent in many parts of the world and guidelines for the use of translators or interpreters has been documented. ${ }^{27}$ Of course, language should not be viewed in isolation from the broader multicultural competencies that need to form significant components of training within mental health facilities. ${ }^{28}$
The finding that three-quarters of the sample did not believe that just one discipline should be responsible for PSR is encouraging. Commenting on the staffing of the PSR treatment mall variation, McLoughlin et al ${ }^{9}$ argue that the programme is not 'owned' by one discipline, emphasizing that it is a multidisciplinary project. The present finding suggests (i) some understanding of the basic premise underlying this therapeutic approach, and (ii) increasing awareness of the need for chronic mental health care to move away from the traditional custodial care systems that were purely medicalised and containment approaches. One of the cornerstones of PSR is that it involves a multidisciplinary approach to care, thus enabling the acquisition of multiple skills and adaptive functions in persons with chronic mental health problem. ${ }^{15}$

Almost $80 \%$ of the participants reported that they do not have sufficient knowledge of PSR practice. On the one hand, this finding raises concern in relation to the current therapeutic interventions that ought to be in place, but the finding may also be suggesting that the mental health professionals are currently using other therapeutic approaches. It must also be remembered that historically mental health policies and training programmes have not focused much on the care of people with chronic mental illnesses, so it is understandable that many mental health workers do not have such skills. On the other hand, the finding is informative, considering that the present investigation was partly aimed at identifying training needs ${ }^{23}$, and is a precursor to a planned PSR training programme. However, it is interesting to note that just over half the respondents stated that their professional training included teaching in PSR. While this may appear at odds with the previous finding it is likely related to the depth of coverage of the topic in basic professional training. Generally PSR is not covered in extensive detail within the basic professional training curriculum of health practitioners (e.g. nurses, psychologists, and others) considering the numerous other core competencies that need to be mastered. A recent US report ${ }^{14}$ also noted that "Except for a very few colleges and universities across the nation, recent graduates do not understand the recovery philosophy and how to implement 
recovery in practice." (p. 297). Clearly the concern over insufficient attention to PSR training is not limited to poorer countries.

That about one-quarter reported receiving in-service training in PSR is positive, but grossly insufficient in the context of the institution's chronic patient load. This deficiency must be viewed against the historical trends in the management of the chronic mentally ill which entailed mainly custodial forms of care rather than an active treatment model. ${ }^{29}$ Again the finding points to a clear motivation to embark on the PSR training programme without delay. It is, therefore, gratifying to note that almost all of the participants believe there should be further training available in PSR, and are keen to undertake such training. These findings are critical in suggesting the presence of appropriate levels of motivation to undertake training which are associated with work commitment, lifelong learning and career development. ${ }^{30}$ The finding that virtually all of the respondents felt that students in their field should have PSR training included in their basic course is certainly one that needs to be taken seriously. In clinical psychology training, for example, competence in the care of chronic mentally ill persons is not included, essentially because the professional training is geared towards care of the acutely mentally disturbed. It is not surprising then that, in the South African context, Ahmed \& Pillay ${ }^{31}$ argued in favour of differently skilling and multi-skilling students so they meet the country's needs. The significant associations noted between aspects of training in PSR and satisfaction with chronic mental health work is not surprising considering the well documented relationship between job training and job satisfaction. ${ }^{32}$ It is also very positive to note this association in this context since work with the chronic mentally ill is rather taxing, with the fruits of the labour slow to emerge.

It is encouraging to note that almost two-thirds of the participants were of the view that the hospital environment lent itself to PSR, although a little over three-quarters of the sample noted that the hospital did not have sufficient resources to implement PSR. These findings must be understood within the context of the institutional issues surrounding the PSR development plans. The authors are aware that while there has been a general keenness by the staff to embark on a PSR programme and the physical environment (albeit needing some improvement) is reasonably conducive, much has been documented about the physical environment needs for rehabilitation programmes. ${ }^{29}$ However, within the institution, there has been much concern over funding for the resources that would be required to adequately implement a PSR intervention. It must be acknowledged that the establishment of an effective programme is not without costs, customised space, fittings and other necessities. ${ }^{9}$ While there has been discussion within the institution about fund raising for the PSR programme, it is also critical that therapeutic interventions such as PSR are awarded the same level of importance as pharmaceuticals which are generally provided without question or suggestion of fund raising. Moreover, there is a need to consider the wider scenario which shows that PSR investments are cost-effective in the long term. ${ }^{11}$ The further finding that only $14 \%$ of participants believed there were sufficient step-down facilities in the community for discharged patients is the unfortunate reality that negatively impacts PSR programmes. The need for supported housing to encourage independent living following discharge from hospital has been documented in the South African context. ${ }^{22}$ The finding that significantly more women than men felt the hospital did not have sufficient resources to do PSR may reflect differences in the way men and women prioritize resources and what they view as significant resources to enable global improvement in functioning. For example, items for personal, domestic and related care have historically received greater attention from women than men. ${ }^{33}$

The finding that $93 \%$ of the participants believed that chronic mentally ill patients can improve in their functioning is suggestive of their confidence in rehabilitation interventions as well as their roles in effecting and facilitating change. This is especially relevant considering staff perceptions of their own effectiveness is related to their actual efficacy and patient recovery. ${ }^{34}$ That a relatively lower percentage (65.8\%) of the respondents felt that these patients could make decisions about their lives may relate to (i) traditional ways of thinking about the chronic mentally ill without the benefit of evidence-based rehabilitation interventions and (ii) the specific types of decisions being contemplated. Since the question was somewhat vague, it is likely that participants may have been considering different levels of decision-making in their responses although rehabilitation research shows very encouraging results in developing decision-making skills such as money management and other life tasks. ${ }^{4}$ Higher level decisionmaking, on the other hand, such as shared decision-making in clinical care still shows this to be an area of challenge. ${ }^{35}$

Most participants (89.5\%) believed that patients' families had a role to play in PSR, which is consistent with the rehabilitation research evidence showing the value of involving families in the treatment programme. ${ }^{4}$ Unfortunately the reality is that very few patients actually have contact with their families, as indicated by the finding that less that $30 \%$ of the respondents noted adequate patient-family contact. Research elsewhere in South Africa also expressed concern about the inadequate patient-family relationships. ${ }^{22}$ The interaction effect showing significantly more men than women believing the patients had sufficient family contact may be explained by (i) the participants thinking about different sets of patients in formulating their responses, or (ii) the historical differences in the way men and women view family and other significant relationships. The former is less likely considering that institution's clinical staff, with the exception of a small number of unit managers (not more than ten), work in rotation across all of the different units and thus have knowledge of virtually all of the patients. The likelihood that the result may be a gendered response to significant relationships must be entertained.

The vast majority of participants were of the opinion that patients with conditions such as mental retardation and chronic schizophrenia could benefit from PSR. This is an encouraging finding, especially when viewed against Liberman' $\mathrm{s}^{36}$ argument (in the context of schizophrenia) that even 'after 30-40 years of active symptoms and disability, some individuals are able to demonstrate improvements that can be tantamount to our definition of recovery" (p.340). Considering the large number of patients in the respondents' institution who have experienced schizophrenia and other chronic conditions for many years, these positive sentiments and could be contribute to overall efficacy in future rehabilitation programmes. ${ }^{34}$

Among the limitations of this investigation, it is acknowledged that a higher response rate could have enhanced the reliability of the findings. Also, it is possible that one or two of the questions could have been interpreted differently by the participants. 


\section{Conclusion}

It is clear that the most of the clinical staff in this investigation do not feel sufficiently equipped to perform PSR interventions, which is not surprising since past mental health policies and training failed to emphasize this component of psychological and psychiatric care. The need for a training programme to address this need is, therefore, evident.

It is, however, encouraging to know that the majority of the staff is satisfied working with the chronic mentally ill, especially considering such job satisfaction can enhance learning motivation in the development of PSR skills. The expressed keenness to undertake training in the area also augurs well for the programme as well as the institution's long-term goal of discharging as many patients as possible to their families and social environments.

\section{References}

1. Smith TE, Liberman RP, Kopelwicz A. Schizophrenic disorders: Rehabilitation. In: Helmchen H, Henn FA, Lauter H, Sartorius N, editors. Current Concepts in Psychiatry Heidelburg: Springer-Verlag, 2000:1-42.

2. Pan L, Mellor D, McCabe M, Hill B, Tan B, Xu Y. An evaluation of the Shanghai Mental Health Service Schizophrenia Rehabilitation Program. American Journal of Psychiatric Rehabilitation 201 1;14:287-306.

3. Davis L, Kurzban S, Brekke J. Self-esteem as a mediator of the relationship between role functioning and symptoms for individuals with severe mental illness: A prospective analysis of Modified Labeling theory. Schizophrenia Research 2012;137:185-189.

4. Kopelowicz A, Wallace CJ, Liberman RP. Psychiatric Rehabilitation. In: Gabbard GO, editor. Treatments of Psychiatric disorders. 4th ed. Arlington, VA: American Psychiatric Publishing Inc, 2007:361-378.

5. Barton R. Psychosocial rehabilitation services in community support systems. Psychiatric Services 1999;50:525-534.

6. Wallace CJ, Liberman RP, Kopelowicz A, Yaeger D. Psychiatric rehabilitation. In: Gabbard GO, editor. Treatment of psychiatric disorders. 3rd ed. Washington, D. C.: American Psychiatric Publishing Co., 2001:1093-1112.

7. World Health Organization. mhGAP Intervention Guide. Geneva: World Health Organization, 2010.

8. Kopelwicz A, Wallace CJ, Liberman RP. Psychiatric Rehabilitation. In: Gabbard GO, editor. Treatments of Psychiatric disorders. 4th ed. Arlington, VA: American Psychiatric Publishing Inc, 2007:361-378.

9. McLoughlin KA, Webb T, Myers M, Skinner K, Adams CH. Developing a psychosocial rehabilitation treatment mall: An implementation model for mental health nurses. Archives of Psychiatric Nursing 2010;24:330-338.

10. Mellsop G, Diesfeld K. Service availability, compulsion, and compulsory hospitalisation. Shanghai Archives of Psychiatry 2012;24:44-45.

11. Liberman RP, Corrigan PW. Implementing and maintaining behavior therapy programs. In: Corrigan PW, Liberman RP, editors. Behavior therapy in psychiatric hospitals. New York: Springer, 1994:201-220.

12. Purgato M, Barbui C, Adams CE. Using the needs of WHO to prioritise Cochrane reviews: The case of antipsychotic drugs. International Journal of Mental Health Systems 201 1;5:25.

13. World Health Organization. Community mental health services will lessen social exclusion. Geneva: World Health Organization, 2007.

14. Olmos-Gallo PA, Starks R, DeRoche Lusczakoski K, Huff S, Mock K. Seven key strategies that work together to create recovery based transformation. Community Mental Health Journal 2012;48:294-301.

15. De Hert M, Detraux J, Peuskens J. Practice of and services for psychosocial rehabilitation of people with schizophrenia in Belgium. Giornale Italiano Di Psicopatologia 2010;16:255-265.

16. Reifels L, Pirkis J. Meso and micro level workforce challenges in psychiatric rehabilitation. Psychiatric Rehabilitation Journal 2012;35:341-343.
17. Yamada A-M, Brekke JS. Addressing mental health disparities through clinical competence not just cultural competence: The need for assessment of sociocultural issues in the delivery of evidence-based psychosocial rehabilitation services. Clinical Psychology Review 2008;28(8):1386-1399

18. Liberman RP, Hilty DM, Drake RE, Tsang HW. Requirements for multidisciplinary team work in psychiatric rehabilitation. Psychiatric Services 2001;52:1331-1342.

19. World Health Organization. The world health report 2001: Mental health: New understanding, new hope. Geneva: World Health Organization, 2001.

20. Mitchell R, Parker V, Giles M, White N. Review: Toward Realizing the Potential of Diversity in Composition of Interprofessional Health Care Teams. Medical Care Research and Review 2010;67(1):3-26.

21. American Psychiatric Association. Report of the Committee to visit South Africa. American Journal of Psychiatry 1979;136:1498-1056.

22. Simpson B, Sambuko T. The psychosocial rehabilitation needs of residents of a half-way house for mental health care users in Durban, South Africa. International Journal of Psychosocial Rehabilitation 2012;16(1):18-28.

23. World Health Organization. Human resources and training in mental health (Mental Health Policy and Service Guidance Package). Geneva: World Health Organization, 2005.

24. McLaughlin K, Muldoon OT, Moutray M. Gender, gender roles and completion of nursing education: A longitudinal study. Nurse Education Today 2010;30(4):303-307.

25. Duffield CM, Roche MA, Blay N, Stasa H. Nursing unit managers, staff retention and the work environment. Journal of Clinical Nursing 2011;20:23-33.

26. Pillay YG, Petersen I. Current practice patterns of clinical and counselling psychologists and their attitudes to transforming mental health policies in South Africa. South African Journal of Psychology 1996;26(76-80.

27. Tribe R, Lane P. Working with interpreters across language and culture in mental health. Journal of Mental Health 2009; 18:233-241.

28. Park-Taylor J, Kin GS, Budianto L, Pfeifer G, Laidlaw P, Sakurai M. Towards reflective practice: A multicultural competence training model from a community mental health centre. Professional Psychology: Research and Practice 2009; 40:88-95.

29. Thorn SH, Bamburg JW, Pittman A. Psychosocial treatment malls for people with intellectual disabilities. Research in Developmental Disabilities 2007;28:531-538.

30. Dirani KM. Professional training as a strategy for staff development: $A$ study in training transfer in the Lebanese context. European Journal of Training and Development 2012;36:158 - 178.

31. Ahmed R, Pillay AL. Reviewing clinical psychology training in the postapartheid period: Have we made any progress? South African Journal of Psychology 2004;34:630-656.

32. Leppel K, Brucker E, Cochran J. The importance of job training to job satisfaction of older workers. Journal Of Aging \& Social Policy 2012;24(1):62-76.

33. Glendinning C. Home care in England: markets in the context of underfunding. Health \& Social Care in the Community 2012;20(3):292-299.

34. Arnold JB. Understanding psychosocial rehabilitation workers' perceptions of difficult psychiatric situations. Unpublished Doctoral Thesis. Walden University, 2011.

35. Matthias MS, Salyers MP, Rollins AL, Frankel RM. Decision making in recovery-oriented mental health care. Psychiatric Rehabilitation Journal 2012;35:304-314

36. Liberman RP. Future directions for research studies and clinical work on recovery from schizophrenia: questions with some answers. International Review of Psychiatry 2002;14:337-342. 